\title{
A Robust Watermarking Scheme for 3D Models based on Encrypted Holographic Algorithm
}

\author{
Mr. Nuthan A $\mathbf{C}^{\mathbf{1}}$, Kiran $\mathrm{S}^{\mathbf{2}}$, Chethan Kumar $\mathrm{C}^{3}$, Prabhuswamy L $\mathbf{4}^{4}$, Ramya $\mathrm{S}^{\mathbf{5}}$ \\ Associate Professor, ECE, GMIT, Mandya, India ${ }^{1}$ \\ $8^{\text {th }}$ Semester, ECE, GMIT, Mandya, India ${ }^{2,3,4,5}$
}

\begin{abstract}
We present a digital watermarking algorithm for 3D model which is based on encrypted holographic digital watermarking algorithm to protect the embedded watermark information (such as a specific identity of the copyright information, etc.) and to improve the security and robustness of the digital watermark information. Firstly, the watermark image is processed by the double random phase modulation to get the hologram watermark which makes a high security. Then in the embedding procedures, affine invariant preprocessing is used for 3D models. We calculate the distance of each vertex to center of gravity of the 3D model which is noted as $r$, and take it as the watermark embedding element to embed the watermark. The experimental results show that the algorithm had the good performance of the watermark robustness to attacks such as noise addition, model simplification, model cropping, and affine attacks. This algorithm can be widely used for digital copyright protection and other aspects of identity hidden.
\end{abstract}

Keywords: Information optics; Holographic algorithm; 3D model; Digital watermarking; Affine invariant; Data confidentiality.

\section{INTRODUCTION}

Three dimensional model has been used in many fields, such as medical industries, movies, video games, constructions and so on. With the growth of application areas, more and more digital products of three-dimensional model spread on the network. The copyright protection of three-dimensional model has become increasingly important. Study on the three dimensional model for digital watermarking technology is becoming a new field of digital watermarking research [1].

Images are the most common cover media for watermarking schemes. With the development of 3D applications, more and more algorithms adopt three-dimensional models as the cover media, either for polygonal models or point geometry. In 1997, Ohbuchi published a paper about the three-dimensional model of digital watermarking [2] in the International Conference which is recognized as the first internationally published article on the three-dimensional model of digital watermarking technology. Research for the copyright protection of three-dimensional model began from that time and got much development in recent years. Among them, the early typical spatial algorithms are mainly Triangle Similarity Quadruple embedding, Tetrahedral Volume Ratio embedding, Mesh Density Pattern embedding [3] etc. However, because those algorithms are simple, they have not enough robustness to be applied to the actual copyright protection. In 2008, Liao put forward a spatial watermarking algorithm for three-dimensional model which is based on the ratio of the distance of focus intersection[4], but the algorithm can not handle mesh simplification and cropping attacks. In 2014, X.Feng proposed two different algorithms to embed watermark into the three-dimensional model [5], but the watermark information is not encrypted. Once the watermarking algorithm is cracked, the watermark information is not safe. In the same year, Wang put forward a digital watermarking algorithm for three-dimensional model which is based on the structural feature of the vertex distribution [6]: the algorithm has the drawbacks that the amount of data embedded is limited. Thereby, this paper applies encrypted holographic watermarking algorithm to the three-dimensional model to protect the watermark information (such as copyright information with specific identity) and improve the security, capacity of watermark embedding.

What's more, the optical holographic transform watermarking algorithm has been successfully applied to the copyright protection of two-dimensional image [7-8]. In our algorithm, the information of embedded watermark can be copyright information and maybe other types of watermark image. We can get the highly similar watermark image even when the model is being attacked.

In our algorithm we choose the distance of barycentre to the vertex on the model as the embedded element noted as $r$ and Hologram information generated from the Optical holographic encryption algorithm as the watermark information. The distance $r$ is affine invariant, so it has good robustness. The Hologram information can be decrypted only by the secret key, so it has highly security. 


\section{IJIREEICE \\ International Journal of Innovative Research in Electrical, Electronics, Instrumentation and Control Engineering \\ ISO 3297:2007 Certified \\ Vol. 5, Issue 6, June 2017}

\section{RELATED WORK}

In 2008, Liao put forward a spatial watermarking algorithm for three-dimensional model which is based on the ratio of the distance of focus intersection [4], but the algorithm cannot handle mesh simplification and cropping attacks.

In 2014, X. Feng proposed two different algorithms to embed watermark into the three-dimensional model [5], but the watermark information is not encrypted. Once the watermark algorithm is cracked, the watermark information is not safe.

In the sameyear, Wang put forward a digital watermarking algorithm for three-dimensional model which is based on the structural feature of the vertex distribution [6], the algorithm has the drawbacks that the amount of data embedded is limited. Thereby, this paper applies encrypted holographic watermarking algorithm to the three-dimensional model to protect the watermark information and improve the security, capacity of watermark embedding.

"Double watermarks of 3D mesh model based on feature segmentation and redundancy information," MultimedTools.

"A watermarking scheme for three-dimensional models by constructing vertex distribution characteristics," Journal of omputer-Aided Design \&Computer Graphics,

\section{III.METHODOLOGY}

\section{A. ENCRYPTION AND DECRYPTION OF HOLOGRAPHIC WATERMARK:}

In order to improve the security of the watermark, we process the watermark with double random phase modulation to generate a holographic watermark. Assume the image or data to be encrypted is the normalized $\mathrm{f}(\mathrm{x}, \mathrm{y})$, and the size

Is $\mathrm{MxN}$. Provided (x,y) is the spatial coordinate, $(\xi, \eta)$ is the coordinate of frequency domain, $\phi(x, y)$ is the image of double random phase encryption, $\mathrm{b}(\xi, \eta)$ is two independent images with random white noise which are uniformly distributed in $[0,1]$. Then we can get:

$\phi(\mathrm{x}, \mathrm{y})=\{\mathrm{f}(\mathrm{x}, \mathrm{y}) \exp [\mathrm{j} 2 \pi \mathrm{p}(\mathrm{x}, \mathrm{y})]\} * \mathrm{~h}(\mathrm{x}, \mathrm{y})$

In the above euation, $\mathrm{h}(\mathrm{x}, \mathrm{y})$ is the Impulse Response of $\mathrm{B}(\xi, \eta)=\exp [\mathrm{j} 2 \pi \mathrm{b}(\xi, \eta)]$, and * means convolution. The decrypted process is the reverse process of the encryption. The encrypted image takes the Fourier transform and then multiplies with $\exp [-\mathrm{j} 2 \pi \mathrm{b}(\xi, \pi)]$, after that takes the inverse Fourier transform and multiplies with $\exp [\mathrm{j} 2 \pi \mathrm{p}(\mathrm{x}, \mathrm{y})]$, then we can get the original image $\mathrm{f}(\mathrm{x}, \mathrm{y})$.Theories prove that $\varphi(\mathrm{x}, \mathrm{y})$ is a white-noise image with mean zero, and it's variance is:

$$
\sigma_{\varphi}^{2}=\frac{1}{M x N}\left[\sum_{u=0}^{M-1} \sum_{v=0}^{N-1} f(u, v)^{2}\right]
$$

\section{B. EMBEDDING ALGORITHMS}

The flow chart of watermark embedding algorithm is shown in Figure 1.The steps are as follows:

1) Process the three-dimensional model with an affine invariant;

2) Sort $\theta_{i}$ of vertices in ascending order, and store the corresponding $\mathrm{r}$ in the matrix $D_{j}$ with size $\mathrm{n} \times \mathrm{n}$, then keep the location information of $\theta_{i}$ in a mark matrix. $\mathrm{j}$ is a positive integer and satisfies $j \leq N /(n x n)+1$, the empty in the last matrix can be filled with zero;

3) Generate random matrix a and matrix b as double random phase modulation, which is key to encryption and decryption of holographic watermark;

4) The watermark image (such as copyright information) modulated by the random phase a takes Fourier transform, then the transformed image is modulated by the random phase $b$ and takes inverse Fourier transform;

5) Coaxial holographic watermark $H$ is then constructed by double random phase encryption;

6) Watermark matrix is expressed as

$$
h_{j}^{\prime}=D_{j}+k \times H
$$

86 where $\mathrm{k}$ indicates the weight of the hologram component. In this case, multiple watermarks are embedded which makes a higher robustness;

7) Three-dimensional Models is reconstructed by matrix $h_{j}^{\prime}$;

8) Spherical coordinates $(r, \theta, \varphi)$ is converted into Euclidean coordinates $(x, y, z)$, and the formula of computation is shown in below 
$x=r \times \sin \theta \times \cos \varphi$

$y=r \times \sin \theta \times \cos \varphi$

$z=r \times \cos \theta$

The hologram algorithm is used successfully in the 3D watermarking.

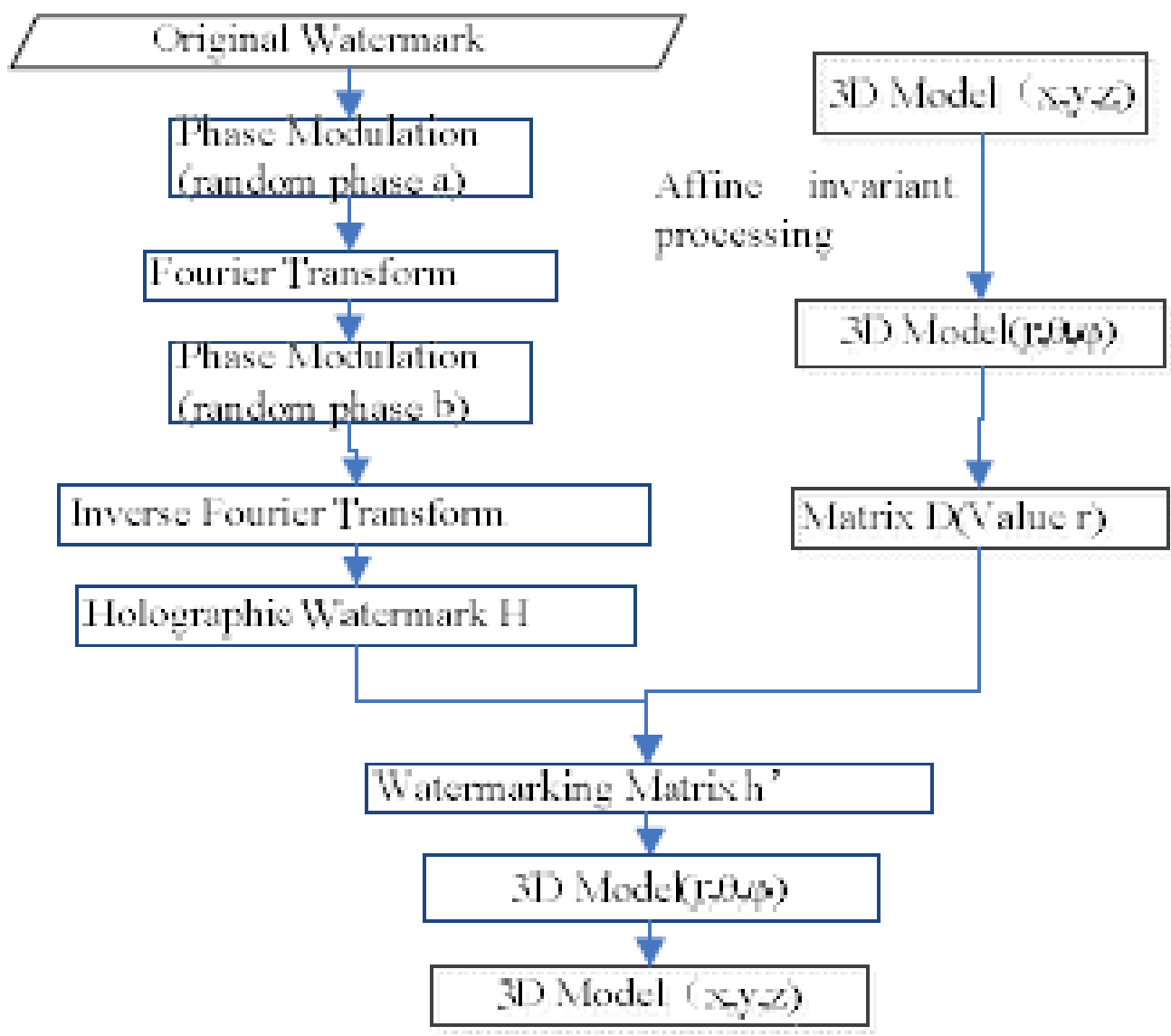

Fig. 1. The basic flowchart of watermark embedding

\section{EXTRACTION ALGORITHMS:}

The flow chart of watermark extraction algorithm is shown in Figure 2.

The steps are as follows:

1) Relocate and Resample operator;

2) Affine invariant processing of three-dimensional model;

3) Move the barycentre of the model to the coordinate origin and Euclidean coordinates (x, y, z) is converted into spherical coordinates $(\mathrm{r}, \theta, \varphi)$;

4) Sort $\theta_{i}$ of vertices in ascending order, and find a matrix with size $\mathrm{n} \times \mathrm{n}$ according to the location of $\theta_{i}$ in mark matrix. If we cannot find the full matrix, then choose the matrix which is relatively integrate and the missing part can be filled with zeros. In this way, hologram watermarking matrix h' is reconstructed (With multiple watermarking embedded, the relatively integrity one is enough);

5) Coaxial holographic watermark $\mathrm{H}$ is then given as $\mathrm{k}$

$$
H=h_{j}^{\prime}-D_{j} / K
$$

6) The holographic watermark $H$ takes Fourier transform. And the transformed information is modulated by the phase $\mathrm{b}$ and takes inverse Fourier transform. Then it is modulated by the random phase -a. And we get the watermark image. 


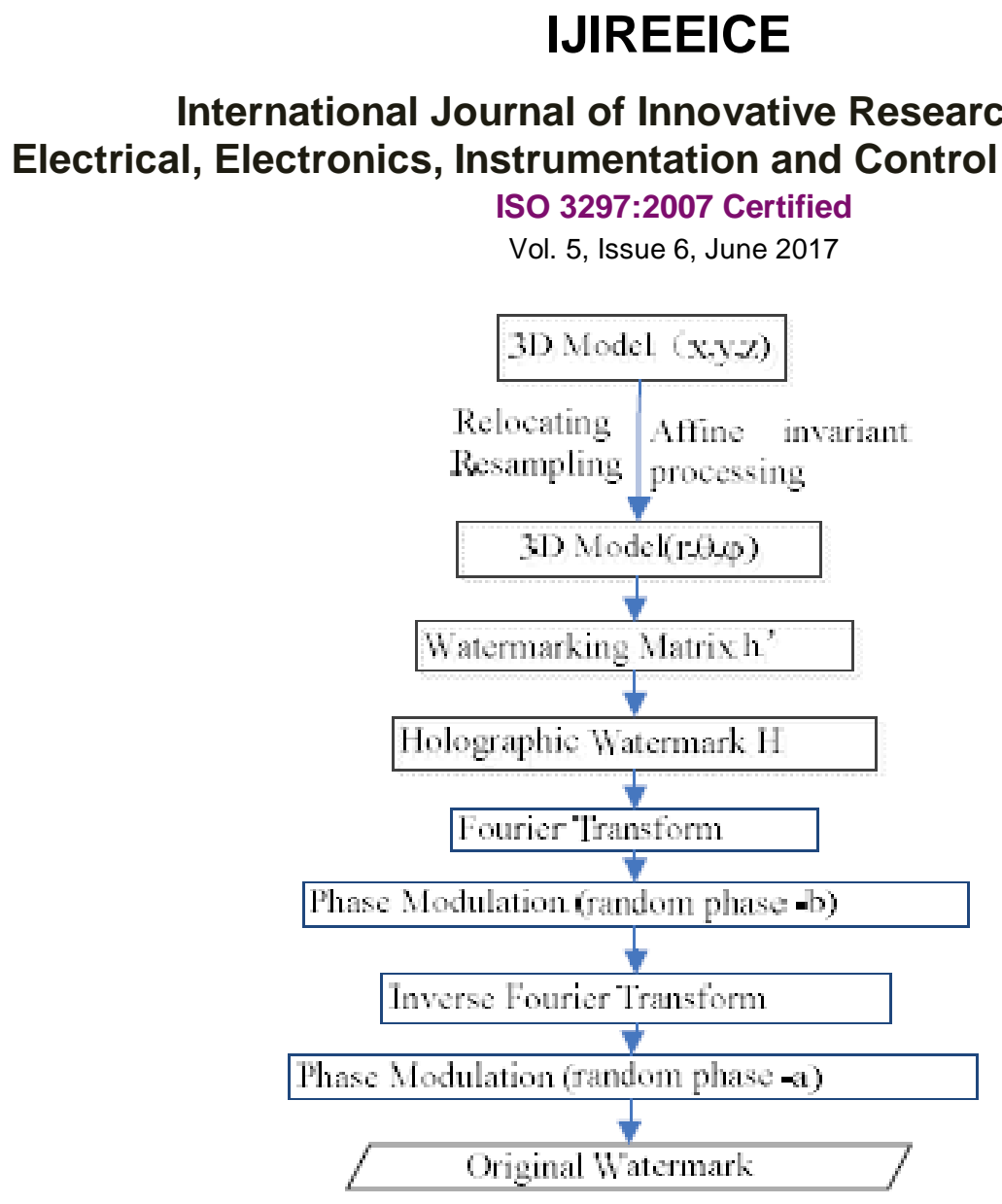

FIG. 2. THE BASIC FLOWCHART OF WATERMARK EXTRACTION

\section{IV.RESULT}

To test the robustness and invisibility of the proposed algorithm, content images are embedded into two different models. One is Bunny, the other is Elephant. The dimension of the watermark image is $(64 \times 64)$, the strength of watermark embedding is 0.001.To prove the generality of the algorithm, we choose different watermark images to embed into the Bunny model and the Elephant model.

In Figure 3, (a) is the original Bunny model, (b) is the watermark image '123', (c) is the embedded Bunny model, (d) is the extracted Holographic watermark image '123', (e) is the original Elephant model, (f) is the watermark image ' $\mathrm{ABC}$ ', (g) is the embedded Elephant model,(h) is the extracted. Holographic watermark image 'ABC'. Through the comparison between (a) and (c), (e) and (g), it proves the invisibility of the watermark. In the followed processing, experiments of noise, simplification, cropping, and rotation are performed.

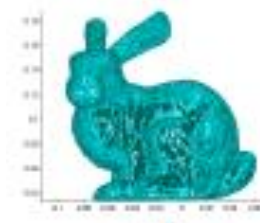

(a)

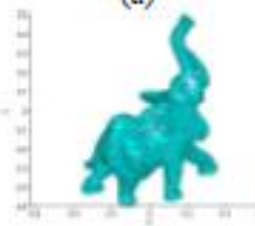

(e)

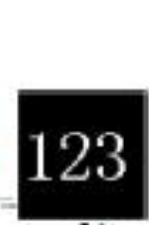

(b)

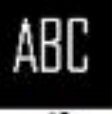

(f)

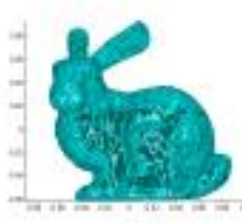

(c)

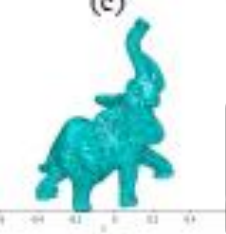

(g)

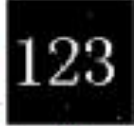

(d)

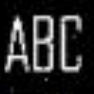

(h)

Fig. 3. (a)(e)Three-dimensional model (b) (f) Watermark image (c) (g)Three dimensional watermark model (d)(h)Decrypted watermark image

\section{A. Noise Attacks}

The experiments of noise attacks are adding the uniform noise into the vertices of the embedded model. The amplitude of noise equals to the results of the mean value of $r$ multiply with the noise intensity $L$. In the experiment we choose different L to prove the good robustness of the algorithm to the noise. The results of experiments are shown in Figure 4. 

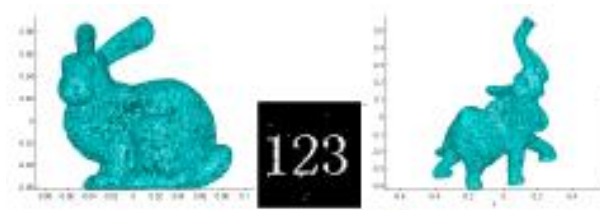

$\mathrm{ABC}$
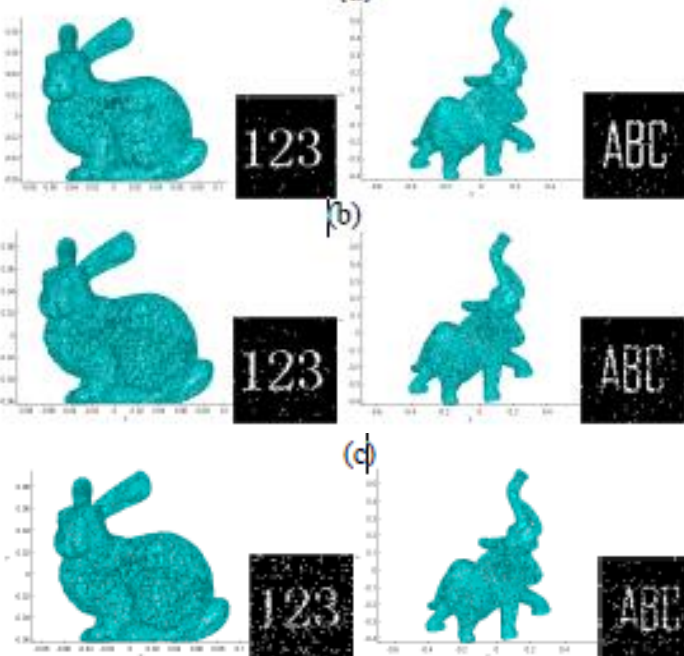

(a)

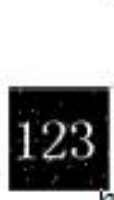

(a)
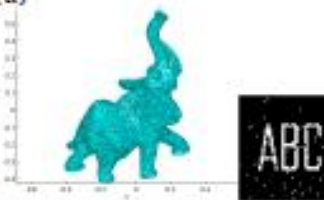

(b)

(c)

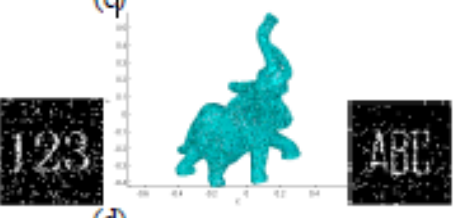

(d)

Fig. 4. (a)Noise $0.1 \%$ (b)Noise $0.3 \%$ (c)Noise $0.5 \%$ (d)Noise $1 \%$

B. Simplification Attacks:

The experiments of simplification attacks are simplifying the vertices of the embedded model. The results of experiments are shown in Table 2 and Figure 5. The experiments prove the good robustness of the algorithm to the simplification.
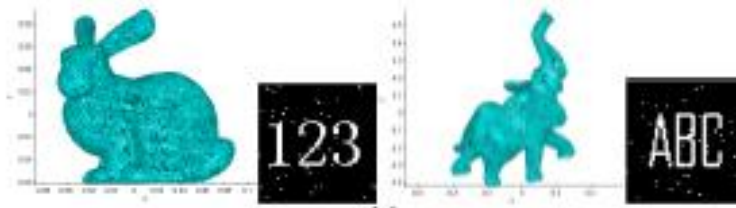

(a)
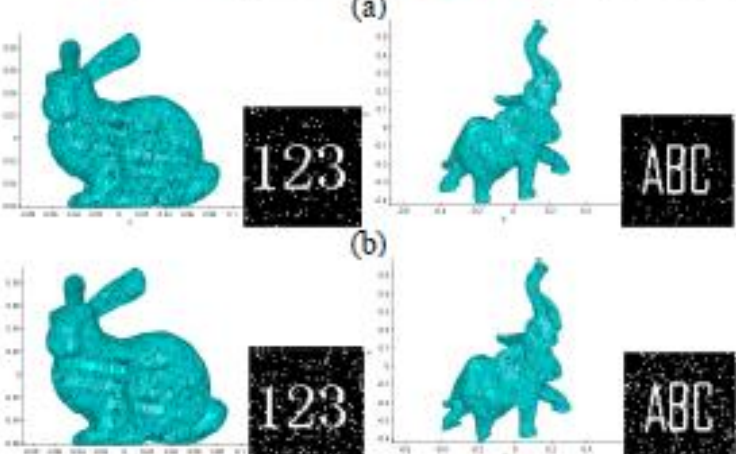

(b)

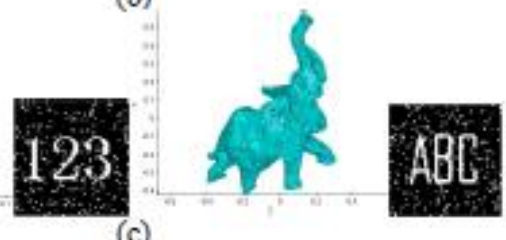

(c)

Fig. 5. (a)Crop30\%(b)Crop86\%(c)Crop40\%(d)Crop60\%

c. Affine attacks:

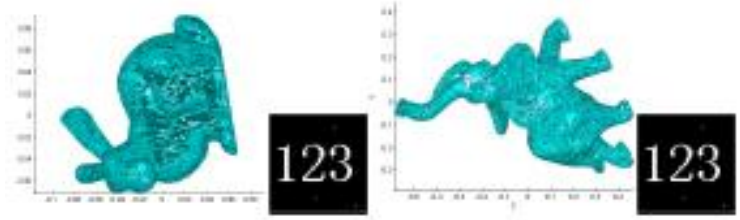

Fig. 6. Rotation attacks 


\section{IJIREEICE

Affine attacks including rotation, translation and uniform scaling attacks are entirely undone by the registration process based on the principal component analysis (PCA). We tested affine robustness against rotation as an example. As expected, our approach achieves good results shown in Figure 6

\section{CONCLUSION}

In this paper we have presented a new robust watermarking for 3D model. This new method is based on encrypted holographic algorithm, using double random phase modulation to make the embedding watermark information more secure. The results clearly show that the presented watermarking procedure can tag objects with watermarks in a robust way. It is invisible and the cropping attacks can be successfully handled. Also the algorithm guarantees the good performance of the watermark robustness to attacks such as addition, model simplification and affine attacks.

\section{REFERENCES}

[1] Min H, Yin X, Liangfeng X, Feng X, “A geometry property based adaptive watermarking Scheme for 3D models,”Journal of Computer- Aided Design \& Computer Graphics, vol.20, pp. 390-402, 2008

[2] Ohbuchi R, Masuda H, Aono M, "Embedding data in 3D models," In Interactive Distributed Multimedia Systems and Telecommunication Services. Springer Berlin Heidelberg, pp. 1-10, January, 1997

[3] Ohbuchi R, Masuda H, Aono M, "Watermarking three dimensional polygonal model through geometric and topological modifications," IEEE Journal on Selected Areas in Communications, vol.16, pp. 551-560, 1998

[4] Xueliang L,Yuping W, "A new spatial domain method for watermarking in 3D models," Chinese Journal of Computers, vol.31, pp.1848-1856, 2008

[5] Xiaoqing F, Wenyu Z, Yanan L, "Double watermarks of 3D mesh model based on feature segmentation and redundancy information," Multimed Tools Appl, vol.68, pp.497-515, 2014

[6] Xinyu W, Yongzhao Z, “A watermarking scheme for three-dimensional models by constructing vertex distribution characteristics," Journal of computer-Aided Design \&Computer Graphics, vol.2, pp.272-279, 2014

[7] Liujie S, Songlin Z, "Digital watermarking of encrypted in-line holography," 0ptics and Precision Engineering, vol.15, pp.428-431,2007

[8] Liujie S, Songlin Z, “Anti-fake technique by double random phase encrypted holographic mark,”A CT A OPT ICA SINICA, vol.27, pp.31- 34, 2007

[9] Chen Y, Medioni G, "Object modeling by registration of mutiple range images," Image and Vision Computing, vol.10, pp.145-155, 1992

\section{BIOGRAPHIES}

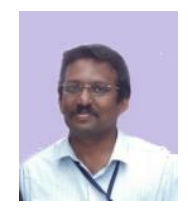

Mr. Nuthan A C Associate Professor, Electronics and Communication Dept., G Madegowda Institute of Technology bharathinagar, Mandya

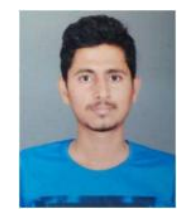

Mr. Kiran S Semester Electronics and Communication Dept., G Madegowda Institute of Technology Bharathinagar, Mandya

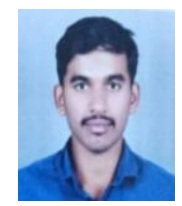

Mr. Chethan Kumar C Semester Electronics and Communication Dept., G Madegowda Institute of Technology Bharathinagar, Mandya

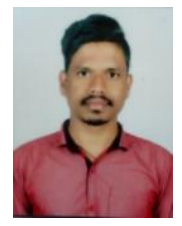

Mr. Prabhuswamy L Semester Electronics and Communication Dept., G Madegowda Institute of Technology Bharathinagar, Mandya

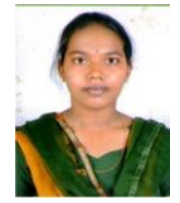

Ms. Ramya S Semester Electronics and Communication Dept., G Madegowda Institute of Technology Bharathinagar, Mandya 